\title{
PROPUESTA METODOLÓGICA ORIENTADA A LA COMPRENSIÓN DE LECTURA
}

\section{A METHODOLOGY FOR READING COMPREHENSION}

\author{
Apolinar Rubén Jara Ascencio \\ Licenciado en Educación Primaria \\ Colegio Maestro Ugo de Censi, Ancash, Perú \\ apolinar_jara@hotmail.com \\ Míriam E. Velázquez Tejeda \\ Doctora en Educación \\ Centro de Investigación USIL, Lima, Perú \\ miriamv2002@yahoo.es
}

\begin{abstract}
Resumen: El tema investigado es la comprensión de lectura por ser el principal problema de aprendizaje de los estudiantes de sexto grado de Primaria. Responde a un llamado del Ministerio de Educación dirigido a realizar propuestas pedagógicas desde el programa de maestría cursado en la Universidad San Ignacio de Loyola 2016. Metodológicamente es una investigación educacional de tipo aplicada. La muestra es intencional y criterial a la que se le aplicaron diferentes métodos e instrumentos como parte del diagnóstico de campo que permitió constatar el estado actual del problema investigado, las causas y las consecuencias en los estudiantes. Como conclusión esencial se modeló una propuesta metodológica que dado sus referentes científicos orienta una transformación en el docente al dirigir el proceso de enseñanza- aprendizaje de la comprensión lectora desde posiciones críticas, reflexivas y metacognitivas que redunde en las formas de pensar, sentir y actuar de los estudiantes.
\end{abstract}

Palabras clave: comprensión de lectura, reflexión, significado, propuesta metodológica.

Abstract: This research is about Reading comprehension as this is sixth grade primary level students' main learning problem. It responds to a request from the Ministry of Education aimed at developing pedagogical suggestions from the master's program at San Ignacio de Loyola University 2016. Methodologically is an applied educational research. The sample used is intentional and judgmental in which we applied different methods and instruments as part of the field diagnostic that allowed us verify the current condition of the researched problem, the reason and the consequences in the students. As an essential conclusion we have shaped a methodological proposal which has scientific references and guides teachers' transformation in directing the reading comprehension learning - teaching process from critical, reflective and metacognitive positions that affects students' way of thinking, feeling and performing.

Key words: reading comprehension, reflection, meaning, and methodological proposal. 


\section{INTRODUCCIÓN}

El siglo XXI ha traído un pujante proceso de globalización unido a grandes cambios en todas las ramas del saber humano, provocando grandes transformaciones y serias diferencias en las personas y en los países a nivel mundial. Para enfrentarse a esos retos se necesita cambiar los sistemas educativos de forma que centren su atención en la formación integral de los estudiantes para que puedan asimilar el volumen de información que se produce, procesarla y producir un conocimiento desde posiciones críticas, valorativas y reflexivas que los preparen para la resolución de problemas de la vida cotidiana.

Ante tal exigencia emerge la lectura como una herramienta esencial para estimular el pensamiento, analizar la conducta humana, adquirir conocimientos y disfrutar del placer que brinda la comprensión de lo leído. La lectura por ser un modelo lingüístico integral le permite al lector ampliar los conocimientos idiomáticos, estimular el pensamiento y el lenguaje, apreciar los valores humanos, desarrollar la capacidad comunicativa tanto oral como por escrito. Al respecto Vargas Llosa, (2007) refirió que, no hay nada tan importante para estimular los sentidos, la pasión y los deseos de las personas como la lectura. En general se reconoce que las personas que han desarrollado la competencia lectora, son reflexivas y saben solucionar los problemas que se le presenten ante la vida.

El problema de la comprensión de lectura ha sido objeto de estudio por diferentes especialistas en el ámbito internacional como es el caso de: Cruzata, (2007), (Bayro y Olivera 2010), Cuellar, (2011), (Camargo y Caro 2011), Ferrer, (2012), (Arrufat y Moya, 2013), Benavides, (2014), Parodi, (2014), (Makuc y Larrañaga 2015) quienes dan fe de diversos enfoques teóricos y han aplicado distinta estrategias didácticas dirigidas a favorecer el desarrollo de las habilidades de comprensión lectora en los estudiantes.

En el campo nacional destaca: Valdez, (2011), Gamonal, (Velázquez y Díaz 2011), (2012), (Espinoza B. y Samaniego 2012, Soto, (2012), Gonzales, (2012), (Fajardo, Lozano, 2013) al afirmar que la comprensión de la lectura es un proceso intelectual que requiere de la estimulación afectiva para que sea una conquista placentera para el lector si es que en la enseñanza se problematiza, analiza, reflexiona sobre las experiencias y las vivencias previas del lector.

Acerca de la comprensión de la lectura, en el Diseño Curricular de Primaria el Ministerio de Educación de Perú, (2009) se precisa que: los estudiantes deben leer y comprender diversos tipos de textos escritos en diferentes situaciones comunicativas mediante los procesos de análisis, interpretación y reflexión que los conduce a la producción de conocimientos significativos; de esta manera se precisa el nivel de eficiencia lectora que se aspira desarrollar en los educandos.

Sin embargo, al observar los resultados de la práctica escolar se constata en los docentes incompetencias en la aplicación de estrategias que contribuyan a un aprendizaje de la lectura como un proceso de análisis crítico y reflexivo de producción de significados, 
de manera que los estudiantes asuman un rol protagónico en el estudio del texto y se potencie el pensamiento crítico al enjuiciar, interpretar, asumir posiciones, predecir y extrapolar el mensaje adquirido a otros contextos de actuación.

Los resultados de las investigaciones de Cruzata, (2007), Cuellar, (2011), Guzmán, (2012), (Fajardo y Lozano 2013) evidencian deficiencias en el desarrollo de las habilidades lectoras, lo cual se corrobora con el diagnóstico aplicado a los estudiantes durante la investigación. Así se identifica una contradicción externa entre el nivel de comprensión de lectura a lograrse según los documentos curriculares del nivel, y las dificultades evidenciadas en las tareas de lectura. Este problema orienta el estudio, cuyo objetivo es conocer las causas y proponer una metodología orientada al desarrollo de la comprensión lectora en los estudiantes de sexto grado de la Educación Primaria.

\section{ANTECEDENTES TEÓRICOS}

A continuación se presentan los fundamentos teóricos y metodológicos que sirven de base epistemológica al estudio de la comprensión de la lectura. Presentación del marco teórico y antecedentes que se presentarán.

\section{Concepciones teóricas que sustentan la importancia de la lectura desde el punto de vista de diversos autores}

Leer es una actividad que potencia la esfera cognitiva y alimenta la espiritualidad. Es un acto intelectual que activa los procesos lógicos del pensamiento a través de la asimilación de la información, procesamiento, asunción de posiciones críticas y producción de nuevos significados que amplían el campo de conocimientos y el desarrollo de las competencias lectoras en general.

La lectura es una herramienta que contribuye a la formación de un lector competente cuando analizada, criticada y reflexionada emplea la experiencia y los saberes previos para solucionar las necesidades y los problemas del entorno socio - cultural donde se desenvuelve. Así lo enfatizan estudiosos del tema como: Roméu, (2004), (Velázquez y Díaz, 2011), (Fajardo y Lozano 2013) y Navarro, (2015), entre otros.

Se comparte el criterio de los especialistas al afirman que leer es un acto comunicativo porque se da a través de un diálogo entre el lector y la información del texto mediante un proceso que activa las experiencias previas y que requiere de la traducción de los símbolos gráficos al campo de las ideas donde se da los juicios de valor como expresa Romèu, (2004). Es en la interacción entre pensamiento y lenguaje donde surgen las incógnitas, las dudas, las predicciones, las hipótesis, las inferencias, los juicios, las críticas, la relación con la realidad de donde emerge la comprensión de lo leído (Benavides y Sierra 2014). 
La principal señal de comprensión de lo leído que alcanza el lector es al darle significado y sentido a lo que lee, al expresar sus ideas en el plano oral o escrito como proceso comunicativo. En tal sentido la lectura deviene en herramienta que potencia el desarrollo de las estructuras cognitivos - afectivos emocionales que ayudan al desarrollo de las capacidades de interpretación y asumir posiciones axiológicas como asevera Makuc, (2015).

Al respecto Dubois, (1986) enfatiza que leer es un proceso de interacción entre pensamiento y lenguaje y la comprensión es la producción del significado por parte del lector. En esa misma perspectiva Solé, (1999) señala que la lectura es un acto de interacción permanente entre el lector y el texto; proceso mediante el cual el primero intenta satisfacer los objetivos que guían su lectura. En línea con ello Benavides, (2014) destaca que la lectura activa el pensamiento, los esquemas previos, las operaciones y las habilidades intelectuales que permiten procesar y arribar a significados de forma auténtica y creativa como enfatiza Ortiz, (2012).

La lectura como actividad de aprendizaje requiere del estudiante una actitud donde cuestione, enjuicie, opine y busque alternativas de solución que son estímulos cognitivos, sociales y culturales que bien tratadas por el docente inciden en la formación integral del educando. Cuando se lee se observa, asimila, analiza, procesa, critica y reflexiona y es a partir de ese tejido de ideas y juicios de donde surgen los significados. Al activarse las habilidades y los saberes previos le otorga una particularidad especial a lo leído.

En relación a ello, (Velázquez y Díaz 2011) enfatizan en que la lectura es un proceso intelectual, que exige concentración durante la percepción, procesamiento de la información, asunción de posiciones, producción y aplicación del conocimiento en la actividad práctica. Es un ejercicio que ejercita el pensamiento, despierta la fantasía, promueve la formación de imágenes, invita a la crítica, a la reflexión y conduce a la autotransformación y a autorregulación.

En ese sentido Solé, (1999), Roméu, (2004), Cassany, (2014) y Navarro, (2015) aseguran que la lectura es una actividad que requiere de una actitud activa, analítica y reflexiva por el lector al enfrentarse a la información del texto, y que al integrarla a su universo cultural brota el nuevo significado personal, fruto del análisis del tejido textual y de las herramientas aplicadas que lo llevan a identificar las acciones, causas, consecuencias, actitudes, hechos, buscar soluciones y extrapolar el mensaje a otros contextos.

\section{La lectura como actividad cultural y formativa}

La lectura es uno de los pilares esenciales que inciden en la formación del ser humano, pues a través de ella se profundiza en la actividad gnoseológica, en la ética humana, la educación ciudadana, se valoran las actitudes y de manera general se amplía el acervo cultural. Es una actividad que conduce a adquirir conocimientos acerca de las 
tradiciones, las costumbres, las formas de vida y relacionarla con la práctica común. Es una vía potenciadora de la formación de los valores identitarios en cualquier sociedad.

Las habilidades de comprensión lectoras, expresa Calero, (2012) se inician con la percepción primaria y en la medida que se analiza se determinan las relaciones causa efecto, los móviles de los actos, las ideas principales y secundarias hasta darle sentido global al texto. En ello interviene la esfera cognitiva, afectiva, motivacional y emocional de la personalidad.

Al respecto Alva, (2008) insiste que la lectura no es solo una actividad cognitiva realizada con el fin de ampliar los horizontes gnoseológicas y culturales del sujeto, es un acto que requiere del apoyo de las vivencias anteriores al comprender el mensaje y solidarizarse con lo ético, lo cual le permite experimentar una satisfacción emocional que lo motiva a su práctica habitual.

La investigación llevada a cabo por (Bayro, y Olivera 2010) demuestra que la lectura es un medio esencial para la formación integral del ser humano para la vida. En realidad la capacidad de recepción de la información, procesamiento y asunción de posiciones permite expresar argumentos y solucionar problemas. Al darle significado ofrece opiniones, critica, valora el comportamiento humano, refuta el mal y asume una actitud progresista que incide en la formación ética de la personalidad.

En línea a ello, Puente, (1996) precisa que el acto de leer hace pensar, analizar y buscar soluciones a los problemas porque activa los conocimientos previos, las habilidades, actitudes axiológicas, la toma de decisiones y el enjuiciamiento de las actitudes de los personajes lo cual es una situación que fortalece las posiciones y posturas críticas ante la vida práctica.

Cassany, (2014) expresó que la lectura crítica es el paso previo al desarrollo de un pensamiento crítico, porque estimula el intelecto al pensar y reflexionar, al interactuar con el texto de forma consciente y autorregulada a través de un diálogo que lo orienta al desarrollo de las habilidades de análisis, predicción, agudización de los sentidos y valoración de los modos de actuación que progresivamente los puede incorporar a su conducta.

\section{La lectura y el proceso de comprensión}

La voz comprender (del latín comprehendere significa entender, penetrar, concebir, discernir, descifrar). Como proceso intelectual la comprensión supone captar los significados que otros han transmitido mediante imágenes, sonidos, colores, movimientos y construir nuevos Roméu, (2004).

Diversos especialistas han conceptualizado la lectura desde diferentes enfoques y perspectivas. Para Goodman, (1998) la lectura es asimilación, interpretación y lo que el lector es capaz de comprender y aprender a través de ella. En ese orden Solé, (1999) afirma 
que leer es relacionar, criticar o superar las ideas expresadas; no implica aceptar tácitamente cualquier proposición, porque exige del que va a criticar $u$ ofrecer otra alternativa, una comprensión cabal de lo que está valorando o cuestionando.

En tal sentido la comprensión como habilidad requiere del dominio de la actividad cognoscitiva, práctica y valorativa porque las acciones del lector dependerán de sus saberes, de las habilidades previas, de la relación dialéctica entre ambas hasta la apropiación del nuevo significado. Es un acto controversial dialógico, de predicciones hasta que se ajusten las estructuras cognitivas donde el propio lector es consciente de lo que aprendió metacognitivamente que influye en su autorregulación al responderse para qué leo, qué conocimientos adquirí, qué me falta por leer, en qué fallé y qué comprendí.

Sobre la autorregulación De Corte, (2015) destaca la importancia de fomentar el aprendizaje autorregulado en los educandos porque cuando estos desarrollan estas habilidades, autorregulan su comportamiento, le dedican tiempo al estudio, se ponen metas que ellos autocontrolan y son más perseverantes a pesar de las dificultades pueden enfrentar al leer un texto, comprenderlo y extrapolar el mensaje a otros contextos.

La comprensión de lo leído exige poner en práctica las dimensiones cognitiva, afectivo- volitiva y emocional del intelecto. Es un acto que estimula el pensar al percibir, procesar y evaluar la información conduciendo a la autosatisfacción por lo leído. Es una actividad en la que las vivencias se entrelazan con el mensaje del texto a través de la interacción entre pensamiento y lenguaje que desencadena en la producción de significados, según Valles, (1998), Zuleta, (2011), (Velázquez y Díaz 2011), (Moore y Narciso, 2011) y Makuc, (2012).

\section{Los niveles de la comprensión de la lectura}

Acerca de los niveles de comprensión de lectura existen diversas concepciones teóricas y metodológicas que interiorizadas por el docente pueden enriquecer su tratamiento didáctico.

Los niveles de comprensión de lectura se conceptualizan como el grado de desarrollo que alcanza el lector durante el estudio del texto, permitiéndole transitar de la percepción primaria a la secundaria al analizar la información, procesarla, evaluarla, producir nuevos significados desde los referentes culturales que posee y aplicarla a la realidad de forma independiente, como asevera Roméu, (2004).

Desde esta posición teórica, se asumen los niveles de comprensión: traducción, interpretación y extrapolación de la especialista, Roméu, (2004) a saber:

Nivel de Traducción. El receptor capta el significado y lo traduce a su código. De forma más sencilla se diría que expresa con sus palabras lo que el texto significa, tanto de manera explícita como implícita, de acuerdo con su universo del saber. Para ello es 
necesario que decodifique el texto, que descubra los intertextos (influencias de otros que revela el lector) y desentrañe el subtexto (significado intencional), según el contexto en el que el texto se produjo.

Nivel de Interpretación. El receptor como resultado del análisis de la percepción secundaria está en condiciones de emitir sus juicios, valoraciones, reflexiones sobre lo que el texto dice y cómo lo dice; asume una posición ante él, opina, compara, evalúa y actúa como lector crítico.

Nivel de Extrapolación. El lector se apropia del contenido del texto, lo comprende y lo aplica o extrapola a otros contextos. Reacciona ante lo leído, infiere y reflexiona. Se autoanaliza, comienza a modificar su conducta y trata de resolver los problemas de su realidad asumiendo así una actitud independiente y creadora que permite la desembocadura del texto sobre otros textos, otros códigos, otros signos, lo que hace al texto intertextual.

La concepción y aplicación de estos niveles permite darle sentido a la lectura y reconstruir el significado del texto. Es un proceso intelectual complejo que requiere de la integración de las ideas de forma globalizadas, permite llegar a la esencia del significado por la activación de los procesos mentales y como expresa Cassany, (2014), es un estímulo para ejercitar el pensamiento crítico y reflexivo necesarios para asumir posiciones ante la vida.

\section{METODOLOGÍA}

La metodología empleada se fundamenta en el enfoque cualitativo educacional de tipo aplicada. En este tipo de investigación el autor parte del conocimiento de su experiencia en el aula, donde identifica la contradicción externa o problema objeto de investigación. Mediante el método científico sistematiza los referentes teóricos que contrasta con la información obtenida a través del diagnóstico de campo y a partir del proceso de triangulación y de aproximaciones sucesivas, logra una comprensión holística del problema, las causas y las consecuencias que inciden en el desarrollo de la comprensión de lectura en los estudiantes. Ante tal situación modela una propuesta metodológica que dados sus fundamentos teórico- didáctico validada por expertos, contribuye a la solución de problema investigado.

\section{Métodos}

Durante el proceso investigativo se emplearon diferentes métodos, técnicas e instrumentos: se utilizaron los métodos teóricos como: el análisis - síntesis para realizar las valoraciones, interpretaciones, reflexiones de las posiciones teóricas, su relación con la problemática y para construir las conclusiones; el inductivo-deductivo se empleó en el estudio epistemológico del problema y el tratamiento actual de los enfoques teóricos; el histórico- lógico permitió acceder a los antecedentes y evolución del campo en el objeto 
para analizarlo desde posiciones interdisciplinarias; el método de modelación hizo posible establecer la relación dialéctica entre la teoría y la práctica para diseñar la propuesta metodológica modelada.

Como técnicas se aplicó la entrevista semiestructurada a 10 docentes del área de Comunicación para conocer el nivel teórico en cuánto a la comprensión de lectura, los niveles de comprensión que conocen, enfoques y estrategias didáctica que aplican en sus clases y qué conocimientos y habilidades poseen sus alumnos; en la prueba aplicada a 25 estudiantes se constataron los conocimientos y las habilidades desarrolladas de los niveles de comprensión y las estrategias de lectura que usan; en la observación a clase a 10 docentes se comprobó el tratamiento teórico y didáctico al dirigir el proceso de aprendizaje de la lectura y el criterio de experto con el fin de analizar, validar la pertinencia científica de la propuesta metodológica según los requisitos que se precisan en la fichas de evaluación.

\section{Los instrumentos aplicados durante a investigación}

Cuestionario de la entrevista semiestructurada a los 10 docentes con el fin de constatar el nivel teórico y metodológico acerca de la categoría investigada; un cuestionario de la prueba pedagógica aplicada a 25 estudiantes para comprobar sus conocimientos y habilidades lectora visto en el dominio de los niveles: traducción, interpretación y extrapolación; guía de observación a clases a 10 docentes con el objetivo de comprobar el proceder teórico y didáctico al dirigir el proceso de aprendizaje, y dos fichas para validar por los expertos la pertinencia científica de la propuesta metodológica.

\section{Procesamiento de los resultados}

En este tipo de investigación Bisquerra, (2005) señala que el autor parte de la identificación de las categorías y subcategorías apriorísticas que son conceptualizadas, sistematizadas y estructuradas en el marco teórico antes de la aplicación de los instrumentos y la recolección de información donde pueden surgir categorías emergentes a partir del análisis, interpretación y triangulación de los resultados obtenidos. El proceso se realizó atendiendo a tres momentos de la investigación:

Primer momento. Reducción de datos y generación de categorías.

Se aplicaron los instrumentos validados por los expertos para su posterior procesamiento de los datos con la ayuda de los métodos cualitativos y cuantitativos, cuyo resultado se analiza por medio de las tablas de reducción de datos y con las de frecuencia. La reducción de datos condujo a conocer el comportamiento de las categorías apriorísticas, las causas, las consecuencias y determinar las tendencias observadas en el proceso de aprendizaje. 
Fig. 1.- Categoría apriorísticas.

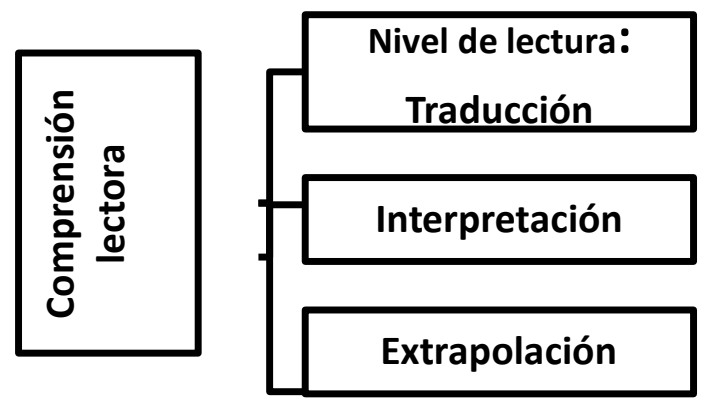

Fuente: Velázquez (2016)

Segundo momento. Se compararon y clasificaron las categorías.

El proceso de triangulación se concretó a partir del análisis de la información aportada por los instrumentos aplicados. El propósito estuvo orientado a generar las conclusiones aproximativas acerca del estado real de la comprensión lectora, a partir de los datos obtenidos por semejanza o contraste. Como resultados del análisis se evidenciaron como categorías emergentes: bajo nivel de lectura: Interpretación y Extrapolación; desmotivación e inadecuado tratamiento didáctico por los docente al proceso de enseñanza- aprendizaje de la comprensión lectora como se refleja en la figura 2.

Fig. 2 Categoría emergentes del proceso de diagnóstico
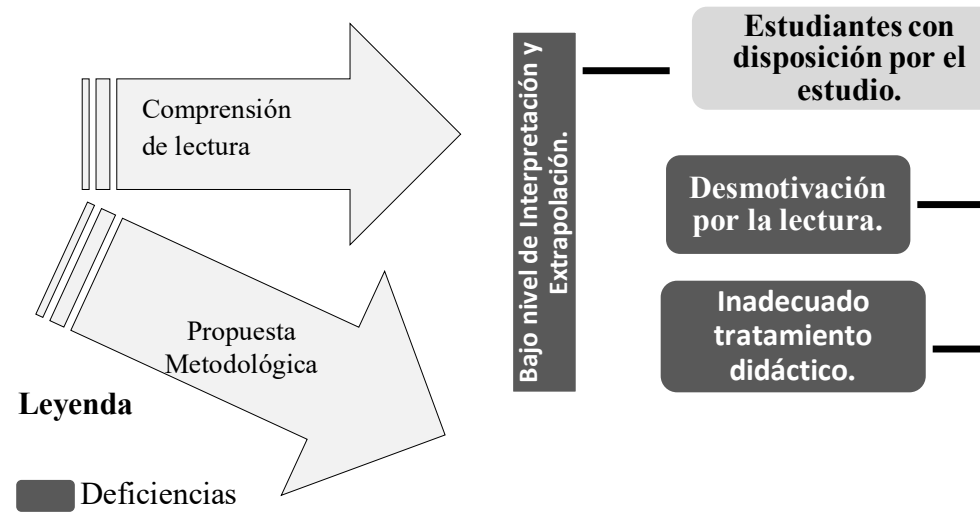

Fortalecer

Fuente: Velázquez (2016)

La prueba pedagógica aplicada a 25 estudiantes se constató que 22 para el $80 \%$, respondieron bien al nivel de lectura Traducción, que es un nivel reproductivo; 6 para el 24 $\%$, responden bien ante el nivel de Interpretación (que, aunque produjeron ideas adecuadas, $19 \%$ educandos evidenciaron falta de conocimientos en sus opiniones, en el análisis y argumentos acerca del tema estudiado que no les permite valorar los hechos, los personajes y dominar los argumentos de la trama del texto), mientras que en el nivel de 
Extrapolación solo 5 estudiantes, para el 20\%, evidenciaron conocimientos y habilidades para realizar la lectura analítica, interpretativa, inferencial y extrapolar la información adquirida a otros contextos de forma creativa, lo que les provoca desmotivación por la lectura, como se observa en la fig. 3.

Fig. 3 Resultado del nivel de comprensión lectora inicial: frecuencia y porcentaje.

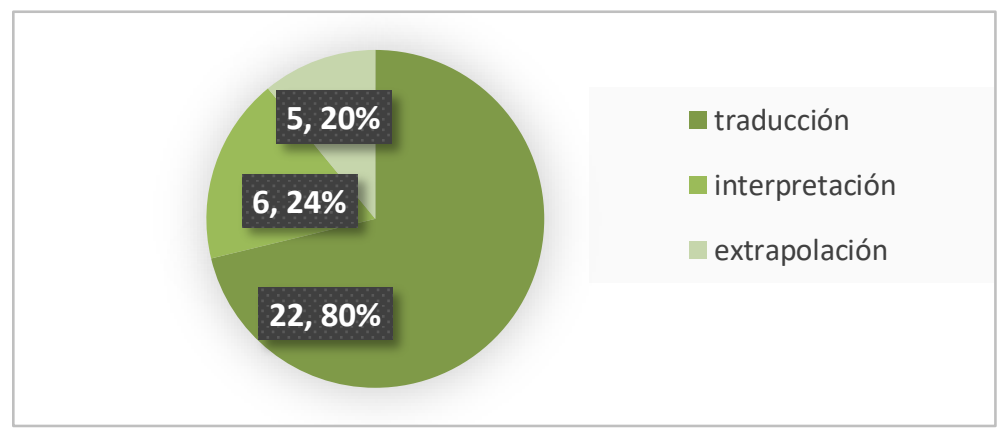

Fuente: Apolinar (2016)

Tercer momento. Interpretación y discusión de los resultados.

Mediante el proceso de triangulación se analizaron las similitudes, las diferencias, las contradicciones, las causas y las consecuencias encontradas desde posiciones dialécticas y holísticas. Estas permitieron llegar a las conclusiones parciales de la investigación como se revela a continuación:

En las entrevistas el $100 \%$ de los docentes manifestaron conocer los enfoques teóricos y didácticos de la lectura como proceso interactivo entre pensamiento y lenguaje, los niveles de comprensión y las estrategia que conduce a la comprensión lectora; sin embargo, se aprecia una contradicción entre el discurso y la práctica pedagógica, pues en las clases observadas evidenciaron deficiencias al conducir el proceso de enseñanza reflejado en la falta de actividades que les permita a los educandos transitar de la dependencia a la independencia cognoscitiva porque los métodos no se orientan a la problematización, al trabajo colaborativo y a la aplicación de dinámicas que fomenten el análisis, inferir ideas, asumir posiciones y aplicar el conocimiento adquirido a otros contextos de la realidad.

Los estudiantes manifiestan un nivel de conocimiento y desarrollo de las habilidades de comprensión lectora inferior a las exigencias de los objetivos curriculares del grado, reflejado en la falta de análisis crítico, interpretación, asunción de posiciones y, en general, no logran resolver actividades del nivel de interpretación y extrapolación de la lectura a otros contextos por falta de ejercitación y de métodos problematizadores, dialógicos y heurísticos en el estudio que les provoca desmotivación por la lectura.

Al valorar los resultados del proceso investigativo se impone la necesidad de diseñar una propuesta metodológica que se oriente a la transformación del proceso de enseñanza- 
aprendizaje de la comprensión lectora y se logren los objetivos de aprendizaje precisados en los documentos curriculares del grado que cursan los estudiantes.

\section{Propuesta metodológica dirigida a la comprensión de lectura}

Como resultado de la sistematización y de los resultados del diagnóstico de campo, la investigación aporta a la práctica pedagógica un producto de orden teórico - práctico consistente en una propuesta metodológica orientada al desarrollo de la comprensión lectora en los estudiantes de sexto de la Educación de Primaria.

La metodología desde el punto de vista psicológico se basa en los referentes teóricos de la escuela Histórico - Cultural de Vygotsky, (1978) al enfatizar que la conciencia es un reflejo de la vida material en la psiquis humana; los fundamentos de la interacción entre pensamiento y lenguaje, la ley de la doble formación de los procesos psicológicos superiores que conlleva a la propiciación consciente del aprendizaje por el sujeto en espacios de socialización y comunicación.

La propuesta diseñada se alimenta de las concepciones teóricas del aprendizaje desde los aportes del enfoque cognitivo, comunicativo y sociocultural de Goodman, (1998), Van Dijk, (2000), Roméu, 2004), la didáctica desarrolladora Castellanos, (2007) y la Neurociencia de Mora, (2014) que se integran dialécticamente con el fin de brindar un tratamiento integral a la lectura como proceso cognoscitivo complejo.

El modelo teórico- funcional representa la dinámica de la propuesta diseñada, que parte del diagnóstico pedagógico para conocer el nivel de desarrollo real de la comprensión lectora de los educandos para dirigir el proceso de aprendizaje desde la didáctica desarrolladora Castellano, (2007) que destaca por la connotación que alcanzan las siguientes categorías didácticas:

La categoría problema se conceptualiza como expresión de las contradicciones del aprendizaje, como punto de partida de su ejecución en la clase, y como condición para potenciar la zona de desarrollo próximo (ZDP) Vygotsky, (1987). Ello precisa conocer las fortalezas y carencias de los educandos para transitar de la dependencia cognoscitiva a la independencia con la mediación del docente y el grupo de iguales. Desde estas perspectivas el problema orienta cómo debe diseñar, organizar, ejecutar y controlar la EnseñanzaAprendizaje para garantizar la eficiencia en las tareas de aprendizaje de los estudiantes de forma que potencien su desarrollo integral.

Los objetivos: se determinan desde el problema, se analizan los más generales como los del grado, los de la asignatura, la unidad, los específicos de la clase y de los estudiantes según su desarrollo actual y potencial. El objetivo es el propósito que el docente y el estudiante son conscientes de ello y cómo proceder para alcanzarlo en la clase al interactuar con la lectura objeto de estudio, de manera que potencie los conocimientos, las habilidades y las actitudes requeridas. 
Fig. 4. Esquema teórico que representa la propuesta diseñada

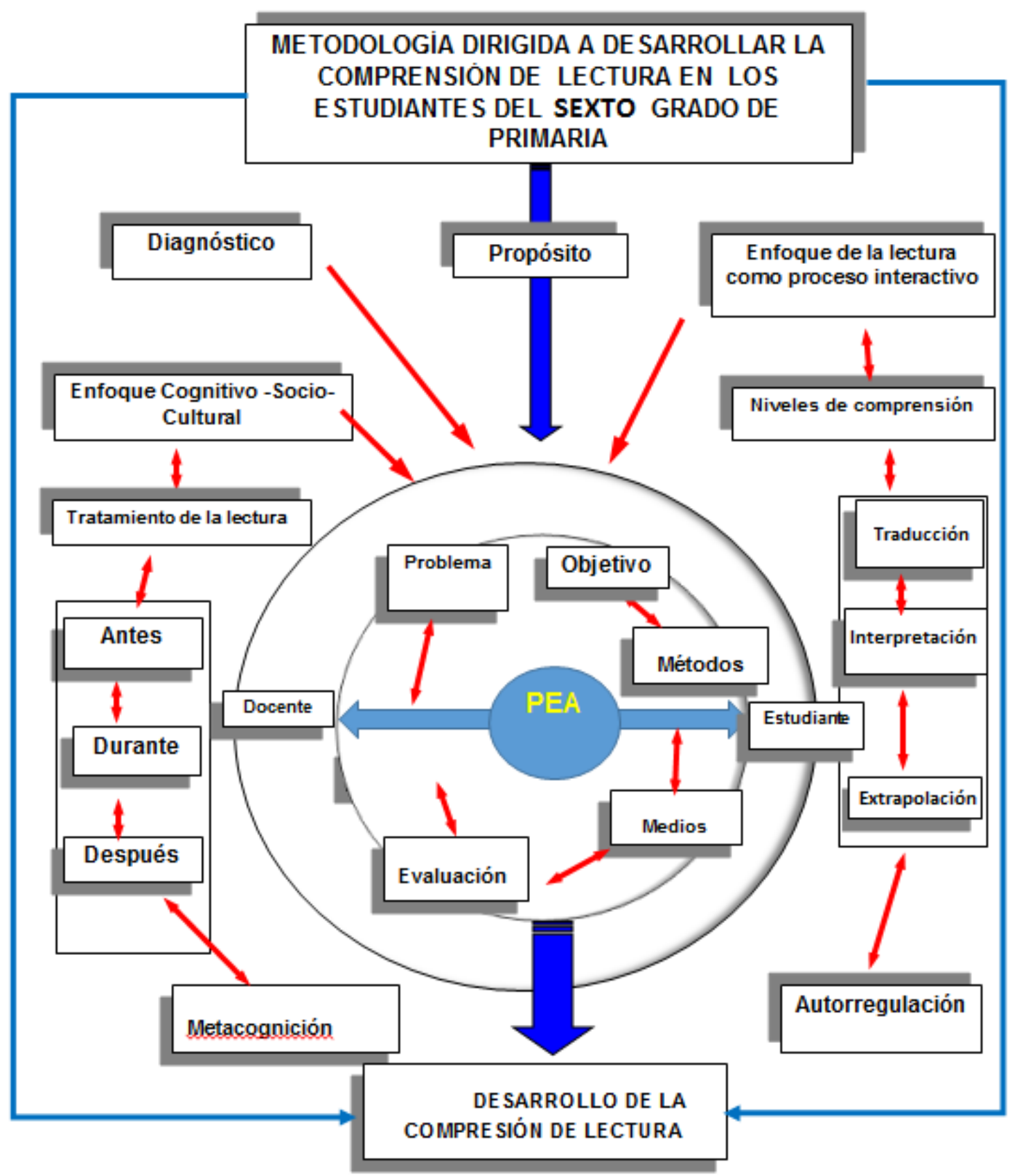

Fuente: Apolinar (2016)

El contenido de la enseñanza: las lecturas son seleccionadas de acuerdo con los objetivos, intereses, las necesidades comunicativas y de comprensión de los estudiantes. Los textos a analizar se deben organizar a partir de las interrogantes: ¿qué lecturas analizar; qué información revela, qué métodos y estrategias emplear, qué valores humanos permite trabajar, cómo estimular la motivación, los intereses, las emociones positivas, qué actividades de extrapolación de lo aprendido pueden realizar? La concepción de la clase se 
orienta a fortalecer las dimensiones: conceptuales, procedimentales y actitudinales de los educandos al interactuar con la lectura.

Los métodos propuestos son problémicos, heurísticos y lúdicos y se concretan a través de una secuencia de actividades de aprendizaje dirigida a lograr los propósitos de la lectura. En su aplicación se atiende la relación lógica que debe existir entre el problemaobjetivo-contenido, priorizando la dinámica del método, pues su relación con el objetivo y el contenido es directa y está condicionado por los flujos dialógicos y dialécticos. Se aplicarán estrategias metacognitivas que orienten la autorregulación de los educandos permitiéndoles detectar logros, dificultades y cómo resolverlas. Se dirigen a lograr tareas de lecturas activas y motivadoras que incidan en el aprendizaje reflexivo al analizarlas desde el autoaprendizaje, autorregulación y la autonomía como enfatizan (Benavides y Sierra 2014).

Las tareas de lectura están graduadas por niveles de complejidad en función del desarrollo alcanzado por los educandos; deben ser motivantes, ejercitarse en las exigencias que demanda el contenido de la lectura y el tipo de texto. El docente debe apoyarse en medios de enseñanza que sirvan de estímulos externos para despertar el interés y la concentración de los educandos al asimilar la información, procesarla, asumir posiciones y despejar las contradicciones dialécticas que requiere la actividad a través de situaciones problemáticas, desafiantes, novedosas y relevantes que propendan al desarrollo de emociones positivas al tener éxito en su realización.

Debe existir un equilibrio entre la exigencia del objetivo, el rigor de la tarea y la posibilidad de realización por el estudiante de acuerdo a su estilo y ritmo de aprendizaje. Estos requisitos didácticos son esenciales para lograr la efectividad en el trabajo con la Zona de Desarrollo Próximo, Vygotsky, (1987). Cuando esta trilogía se integra y son atendidas didácticamente se crean condiciones excepcionales que redundan en la comprensión de lectura. Al estar conscientes de lo que aprenden y tener los recursos de cómo hacerlo, provoca en ellos niveles de motivación intrínseca porque se saben orientados y se incentiva el interés y la concentración en las acciones a desplegar para comprender lo leído, lo cual fortalece la autoconfianza, la autoestima y el autoconocimiento porque encuentra satisfacción en el aprendizaje.

La evaluación se concibe como parte del proceso de aprendizaje en sus formas diversas como: diagnóstica, la formativa y la sumativa. El contenido objeto de evaluación se orienta a los aspectos conceptuales desde la información del texto, lo procedimental al interactuar con los diferentes tipos de tareas de lectura y lo actitudinal se trabaja a partir de la valoración ética que realizan. Ello necesita de instrumentos que orienten al educando al qué se evalúa, cómo, para qué y qué instrumentos se aplicarán en la clase como: registros anecdóticos, rúbricas, listado de cotejo, mapas conceptuales, los diarios de clase, los textos escritos, la participación en las dramatizaciones, las entrevistas, los diálogos, debates y asambleas, entre otras. 
Se propone distintos tipos de clases según la función didáctica como: clase de presentación de lectura, clase para ejercitar las habilidades lectoras y la clase de lectura creadora con el festival de la lectura, cuyo propósito es demostrar los conocimientos y las habilidades desarrolladas y fomentar el interés por la lectura. Se potencia el trabajo individual y grupal como premisa para el estudio del texto mediante la colaboración, el diálogo, reflexión y la convivencia democrática. El festival de la lectura a nivel de escuela se realizará una vez al mes y en ella, los estudiantes expondrán lo aprendido en las lecturas a través de actividades como: producción de textos, mesa redonda, paneles informativos, exposiciones, charlas, conferencias, concursos y dramatizaciones donde podrán dar a conocer: autores, lecturas, personajes aplicando toda la creatividad que los docentes y estudiantes pueden llevar a la práctica.

El rol de los estudiantes es protagónico y activo, construyen y reconstruyen el mensaje de la lectura; autorregulan su actividad de estudio en el contexto del grupo, interactúan e intercambian puntos de vistas y asumen posiciones. El análisis de la lectura se organiza en comunidades de colaboración, diálogo, reflexión y ayuda mutua. El trabajo en equipo exige organizarse, cooperar y socializar ideas lo que permite clarificar conceptos, resolver problemas de forma conjunta y potenciar el fomento de la convivencia democrática en el aula.

El rol del docente es de mediador eficiente, cuya función es propiciar un aprendizaje consciente y ofrecer los niveles de ayuda requeridos para promover el tránsito gradual de los niveles de desarrollo inferiores a superiores (ZDP), Vygotsky, (1978). Debe crear ambientes de seguridad, empatía y estados emocionales positivos al aplicar métodos creativos, lúdicos y heurístico que provoquen la extrañeza y lo novedoso a lo habitual para que el aprendizaje resulte un paraje de alegría y satisfacción como refiere Mora, (2014), al enfatizar que cuando se aprende en un ambiente de alegría y satisfacción, se logra un aprendizaje sólido y duradero.

\section{Valoración de la propuesta metodológica por los especialistas}

Para validar la efectividad de la propuesta metodológica modelada se empleó el método de criterio de expertos con dos fichas de valoración de aspectos externo e interno del producto científico. Los especialistas fueron elegidos a partir de los siguientes criterios: poseer el grado de Maestro o Doctor en educación, haberse desempeñado como docente de Primaria y tener experiencia en la investigación científica.

En el proceso de la validación cuantitativa se codificaron diez criterios en las fichas, donde señalaron el valor que asignaron a la propuesta a partir del puntaje: 1 deficiente, 2 bajo, 3 regular, 4 buena y 5 muy buena. La validación también se llevó a cabo desde el punto de vista cualitativo a través de los indicadores: positivos, negativos y sugerencias si fuera necesario. Los criterios a valorar en la propuesta en su aspecto externo fueron: claridad, objetividad, actualidad, organización, suficiencia, intencionalidad, consistencia, coherencia, metodología y pertinencia. 
Fig.5. Valoración interna y externa de la propuesta

\begin{tabular}{|l|c|}
\hline \multicolumn{1}{|c|}{ Escala } & Valoración \\
\hline $0-25$ & Deficiente \\
\hline $26-59$ & Baja \\
\hline $60-70$ & Regular \\
\hline $71-90$ & Buena \\
\hline $91-100$ & Muy buena \\
\hline
\end{tabular}

Fuente: Apolinar (2016)

Los resultados finales de la valoración ofrecida por los especialistas se expresan a continuación:

Fig.6. Valoración de los resultados

\begin{tabular}{|c|c|c|}
\hline $\begin{array}{c}\text { Sumatoria de } \\
\text { valoración total }\end{array}$ & $\begin{array}{c}\text { Promedio de } \\
\text { valoración }\end{array}$ & Valoración \\
\hline 176 & 88 & Bueno \\
\hline \multicolumn{3}{|c|}{ Fuente: Apolinar (2016) } \\
\hline
\end{tabular}

La evaluación de los especialistas al producto presentado es buena, precisan que procede la aplicación de la propuesta metodológica para el nivel educacional indicado. Reconocen que los fundamentos científicos, teóricos y didácticos que la asisten y su carácter flexible y creativo permiten conocer que existen las condiciones para su posible generalización en otros grados de la Educación Primaria, teniendo en cuenta las características psicopedagógicas de los estudiantes y del contexto donde se pretenda aplicar.

\section{CONCLUSIONES}

El proceso investigativo demuestra que el desarrollo de la comprensión lectora implica por parte del docente un eficiente trabajo didáctico e individualizado a partir de que cada estudiante tiene sus propias experiencias, estilos, ritmos, piensa y aprende de forma diferente de ahí que la actividad de lectura debe orientarse a potenciar su capacidad de análisis mediante la interacción, socialización y autorreflexión permanente para lograr los niveles de desarrollo de comprensión lectora requeridos.

El aporte esencial de la investigación es de carácter teórico- práctico al brindar a la comunidad pedagógica una propuesta metodológica que toma en cuenta el nivel de desarrollo actual del aprendizaje en lo cognitivo, afectivo y creativo de los educandos con el fin de promoverlos a niveles superiores de desarrollo durante el proceso de comprensión de lectura que en manos de los docentes se convierte en una guía que les orienta a planificar, dirigir y evaluar el aprendizaje de la comprensión lectora.

La aplicación de la prueba de salida aplicada a los 25 estudiantes demostró un avance en la comprensión lectora en relación a la inicial, al ubicarse 24 en el nivel de 
Traducción para el $96 \%$ de respuestas correctas; 21 en el nivel de Interpretación para el $84 \%$ de avances en las habilidades lectoras y 20 respondieron bien ante las tareas de Extrapolación para el $88 \%$ pudiendo aplicar el mensaje en otros contextos con creatividad. Este resultado a pesar de haberse obtenido a modo de pilotaje en un trimestre, da cuenta de la efectividad de la metodología vista en el desarrollo de las habilidades de comprensión lectora alcanzada por los estudiantes como se observa en la fig.7.

Fig. 7 Resultados del nivel de comprensión lectora inicial y final, en frecuencia y porcentaje.

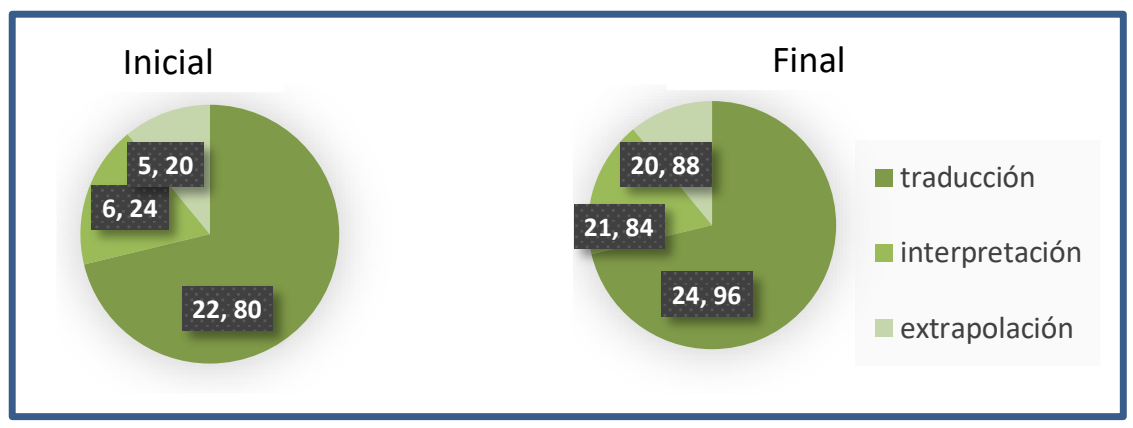

Fuente: Velázquez (2016).

Las actividades desarrolladas como parte de la metodología que más incidieron en que los estudiantes experimentaran un salto cualitativo superior, fue escribir textos libres sobre lo leído, exponer sus ideas en los paneles informativos y en las dramatizaciones de lectura que además de potenciar la comprensión lectora en sí, permitieron enriquecer las habilidades comunicativas y la formación de valores al analizar la conducta de los personajes.

Se hace necesario validar el impacto de la aplicación de la propuesta metodológica en una muestra más numerosa a fin de comprobar su contribución al desarrollo de la comprensión lectora desde las exigencias de un proceso de enseñanza-aprendizaje donde el docente asume un desempeño profesional competente, de manera que le permita al equipo de investigadores hacer los ajustes y continuar el estudio en otras aristas del tema que también inciden en la formación integral del estudiantado desde la lectura.

\section{REFERENCIAS BIBLIOGRÁFICAS}

Alva, S. (2008) Metodología de la investigación. http://www.scribd.com/doc/8921 46/moduloinvestigadaaplicada

Bayro, A.; Olivera M. (2010) La comprensión lectora y la utilización de estrategias de organización en los alumnos con dificultades de aprendizaje del nivel secundario del colegio Antares de Surco. Tesis de maestría publicada. Pontificia Universidad Católica del Perú, Lima, Perú. 
Benavides, R., Sierra, G. (2014) Estrategias didácticas para fomentar la lectura crítica desde la perspectiva de la transversalidad. REICE. Revista Iberoamericana sobre Calidad, Eficacia y Cambio en Educación 11(3): 79-109.

Bisquerra, R. (2005) Métodos de la investigación educativa. Guía práctica. España: CEAC.

Bueno, B. (2015) Neurociencia y Educación. http://www.oei.es/divulgacioncientifica/entrevistas 142.htm

Cáceres, A., Donoso, P. Guzmán. J. (2012) La comprensión lectora Significado que le atribuye las/ los docentes al proceso de comprensión lectora en NB. Tesis para optar el grado de Maestría. Chile.

Castellanos D. (2007). Reflexiones metacognitivas y estrategias de aprendizaje. La Habana: ISP Enrique José Varona.

Cassany, D. (2014) La lectura crítica. Barcelona: Editorial Graó.

Calero, A. (2012) Cómo mejorar la comprensión lectora. Madrid: Wolters Kluwer.

Camargo, Z., Uribe, G. y Caro, M. (2011) Didáctica de la comprensión y producción de textos académicos. Armenia: Publicaciones Universidad del Quindío.

Cruzata, A. (2007) Estrategia didáctica para el tratamiento a la competencia literaria: percepción y producción crítica de textos literarios en la educación preuniversitaria. Tesis para optar el grado de Doctor en Ciencias Pedagógicas.

De Corte E. (2015) Aprendizaje constructivo, autorregulado, situado y colaborativo: un acercamiento a la adquisición de la competencia adaptativa (matemática). Revista Páginas de Educación 8(2) http://revistas.ucu.edu.uy/index.php/paginasdeeducacion/article/view/690

Dubois (1995) The Souls of Black Folk. New York: Penguin Books.

Logan, R. (1971) W.E.B. DuBois. New York: Hill and Wang. Marable.

Goodman (1998). El proceso de la lectura: consideraciones a través de las lenguas y del desarrollo. México.

Gonzales, M. (2012) Teorías implícitas de docentes en el área de comunicación, comprensión lectora. Tesis para optar el grado de Maestría en Educación. Pontificia Universidad Católica del Perú.

Makuc, M. (2015) Teorías implícitas de los profesores acerca de la comprensión de textos. Punta Arenas: Universidad de Magallanes.

Ministerio de Educación (2009) Diseño Curricular Nacional de Educación Básica Regular. Lima, Perú.

Mora, F. (2014) Neuroeducación. Madrid: Alianza Editorial. 
Navarro (2015) El efecto del aprendizaje activo en el desarrollo de habilidades propias del pensamiento crítico en estudiantes de ciencias. Revista de Psicodidáctica 20 (2): 209226. http://www.ehu.eus/revista-psicodidactica.

Ortiz, M. (2012) Influencia de los mapas conceptuales en el rendimiento académico segundo grado del nivel secundario del colegio experimental de aplicación de la Universidad Nacional de Educación Enrique Guzmán.

Solé, I. (1999) Estrategias de lectura. Barcelona, España.

Soto, I. Espinoza, B. y Masaniego, D. (2012) Componente sintáctico del lenguaje oral y la compresión lectora en niños de 10 y 11 años de Instituciones Educativas particulares y estatales del distrito de breña de Lima. Pontificia Universidad Católica del Perú. Lima.

Parodi, G. (2014) Comprensión de textos escritos. La teoría de la comunicabilidad. Buenos Aires: EUDEBA.

Puente, A. (1999) El cerebro creador. ¿Qué hacer para que el cerebro sea eficaz? Madrid: Alianza.

Roméu, A. (2004). El enfoque cognitivo, comunicativo y de orientación sociocultural. Dimensiones e indicadores de la competencia comunicativa y sociocultural. Cuidad de la Habana: Pueblo y Educación.

VanDijk, T. (2000) El discurso como estructura y proceso. Estudios sobre el discurso l. Barcelona: Gedisa.

Valdez, J. (2011) Estrategias de aprendizaje, comprensión lectora y rendimiento académico de los estudiantes de la signatura de didáctica del lenguaje 1. Tesis para optar el grado de Maestría. Universidad Nacional La Cantuta. Lima, Perú.

Valles, A. (1998) Compresión lectora y proceso psicológicos. España.

Zuleta, E. (2011) Sobre la lectura fotocopioteca. http://www.lugaradudas.org/publicaciones/fotocopioteca/12 juana anzellini.pdf. 